\title{
Enzymaktivitäten in verschiedenen Rattenorganen nach Verfütterung einer Mg-Mangel-Kost
}

\author{
Von Th. GüNTHER \\ Aus dem Physiologisch-Chemischen Institut der Freien Universität Berlin (Direktor: Prof. Dr. Dr. E. Scbütte)
}

(Eingegangen am 29. September 1969)

Nach 38tägiger. Mg-armer Ernährung waren bei Ratten in der Leber dic Aktivitäten von Glucrsc-6-phosphatdchydrogenasc, Enolase, Pyruvatkinase sowic Aspartattransaminase und im Herz dic Aktivitäten von Glucose-6-phosphatdehydrogenase und Pyruvatkinase crhöht. Im Skelettmuskcl war dic Aktivität der Glucuse-6-phosphathydrogenase angestiegen, dic vonn Aldolase, Enoslase und Pyruvatkinase vermindert. Im Scrum hatte die Aktivität der Pyruvatkinase und der Aspartattransaminase zugenommen, dic der Enolase abgenommen.

\section{Enzyme activities in various organs of the rat after feeding a Mg-deficient diet}

Rats wcre fed for 38 days on a $\mathrm{Mg}$-deficient diet. The activities of glucose-6-phosphate dehydrogenase, enolase, pyruvate kinase and aspartate transaminase in the liver, and glucose-6-phosphate dehydrogenase and pyruvate kinase in the heart were increased. In skeletal muscle, the activity of glucose-6-phosphate dehydrogenase increased, whilc aldolase, enolase and pyruvate kinase showed a decreased activity. In the scrum, pyruvate kinase and aspartate transaminase were increased and enolase was decreased.

Bei der Analyse der Stoffwechselveränderungen, die bei $\mathrm{Mg}$-verarmten Zellen von $E$. coli auftreten, fanden wir, $\mathrm{da} ß$ in den $\mathrm{Mg}$-verarmten Zellen verschiedene Enzyme in veränderter Konzentration vorliegen $(1,2) . \mathrm{Da}$ in der Leber und Muskulatur von Mg-arm ernährten Ratten gleichartige Änderungen im Wasser- und K-Gehalt wie bei $\mathrm{Mg}$-arm gewachsenen Zellen von E. coli auftreten (3), untersuchten wir auch den Enzymgehalt einiger Rattenorgane.

\section{Methode}

Die Ratten wurden, wic in der vorangegangenen Mitteilung beschricben (3), behandelt.

Die in Äthernarkose entnommenen identischen Organproben wurden sofort in flüssiger Luft eingefroren und bei $-20^{\circ}$ aufbewahrt. Zur Bestimmung der Enzymaktivitäten wurden dic Organe gewogen. Etwa $1 \mathrm{~g}$ wurde mit $7 \mathrm{~m} / 0,25 \mathrm{M}$ Rohrzuckerlösung, dic $2 \mathrm{~mm} \mathrm{MgCl}_{2}$ und $2 \mathrm{~mm}$ EDTA enthielt und auf $\mathrm{pH} 7,6$ eingestellt war, im Ultraturrax $5 \mathrm{mal} \mathrm{je} 1 / 4 \mathrm{Min}$. homogenisiert und dazwischen jeweils $3 / 4 \mathrm{Min}$. in ciner Eis-NaCl-Mischung gekühlt und anschließend noch etwa $1 / 4 \mathrm{Min}$. mit Ultraschall unter Kühlen nachbehandelt.

Die Enzymaktivitäten im $40000-g$-Uberstand wurden bei $25^{\circ}$ in optischen Testen bestimmt. Dic Ansätze in $2 \mathrm{~m} / 0,075 \mathrm{M}$ Triäthanolaminpuffer $\mathrm{pH} 7,6$ cnthiclten (in $\mu \mathrm{Mol}$ ):

HEX ${ }^{1}$ ): Glucosc 4, $\mathrm{MgCl}_{2}$ 2, NADP 1, ATP 1, G-6-PDH $20 \mu \mathrm{g}$, Staxt mit ATP.

PFK: $\quad \mathrm{MgCl}_{2}$ 5, ATP 0,4, NADH 0,3, Fructosc-6-phosphat (F-6-P) 1,3, ALD $\left.{ }^{1}\right) 200 \mu \mathrm{g}$, Glyccrophosphatdehydrogenase (GDH) $20 \mu \mathrm{g}$, Trioscisomerase (TIM) $20 \mu \mathrm{g}$, Start mit F-6-P.

1) Ablirzungen: HEX: Hexokinase (EC 2.7.1.1); PFK: Phosphofruktokinase (EC 2.7.1.11); ALD: Aldolase (EC 4.1.2.13); ENOJ.: Enolase (EC 4.2.1.11); PK: Pyruvatkinase (EC 2.7.1.40); LDH: Lactatdehydrogenase (EC 1.1.1.27); G-6-PDH: Glucosc-6-phessphatdehydrogenase (EC 1.1.1.40); GLDH: Glutamatdchydrogenase (EC 1.4.1.2); GP'T: Glutamat-pyruvat-transaminase (EC 2.6.1.1); GOT:: Glutamat-oxalacetat-transaminasc (EC 2.6.1.1); MDH: Malatdehydrogenase (EC 1.1.1.37); ICDH: Isozitratdchydrogenase (EC 1.1.1.42).
ALD: NADH 0,3, EDTA 4, $\mathrm{MgCl}_{2} 4$, Pructosc-1,6-diphosphat (FDP) 8, GDH $20 \mu \mathrm{g}$, TIM $20 \mu \mathrm{g}$, Start mit PDP.

ENOL: $\quad \mathrm{MgCl}_{2} 10, \mathrm{KCl} 150, \mathrm{NADI} / \mathrm{0}, 3,2$-Phosphoglyccrinsäurc 1,2, ADP 2, PK $200 \mu \mathrm{g}$, JDI $100 \mu \mathrm{g}$, Start mit 2-l'hosphoglyccrinsäure.

PK: NADH 0,3, ADP 2, Phosphoenolpyruvat $1,6, \mathrm{MgCl}_{2}$ $10, \mathrm{KCl} 200$, IDH $100 \mu \mathrm{g}$, Start mit ADP.

LDH: NADH 0,3, Pyruvat 4,8, $\mathrm{MgCl}_{2}$ 4, EDTA 4, Start mit Pyruvat.

G-6-PDH: $\mathrm{MgCl}_{2}$ 4, EDTA 4, NADP 0,3, Glucose-6-phosphat (G-6-P) 3,6, Start mit G-6-P.

GLDH: 2-Oxroglutarsäure 28, $\mathrm{NH}_{4} \mathrm{Cl} 100$, NADH $0,3, \mathrm{MgCl}_{2}$ 4, EDT'A 4, Start mit 2-Oxoglutarsäurc.

GP'T: NADH 0,3, D,L-Alanin 40, 2-Oxoglutarat 20, LDH $100 \mathrm{~kg}$, Start mit 2-Oxoglutarat.

GOT: 2-Oxoglutarsäure 20, s-Asparaginat 105, NADH 0,3, MDH $100 / \mathrm{gg}$, Start mit 2-Oxoglutarsäure.

ICDH: $\mathrm{MgCl}_{2}$ 28, EDTA 4, NADP 0,3, D-Isocitrat 2,6, Start mit D-Isocitrat.

\section{Ergebnisse und Diskussion}

Die Enzymaktivitäten der einzelnen Organe sind in Tabelle 1 dargestellt. Die gemessenen Enzymaktivitäten stimmen überein mit den Enzymaktivitäten anderer Untersucher (4) mit Ausnahme der Aktivitäten von Hexokinase und Phosphofruktokinase. Bei diesen Enzymen fanden wir wesentlich geringere Aktivitäten. Sie liegen in der Größenurdnung, die LONG (5) an der Leber ermittelt hat. Diese geringeren Aktivitäten der Hexokinase und Phosphofruktokinase sind wahrscheinlich bedingt durch die intensivere Homogenisierung im Ultraturrax und mit Ultraschall gegenüber dem Aufschluß im Potter-Elvehjem-Homogenisator.

Von den untersuchten En\%ymen war dic Glucosc-6phosphatdehydrogenase in den Organen der Mg-arm ernährten Ratten erhöht. Dic größste Zunahme fanden 
Tab. 1

Enzymaktivitäten in verschiedenen Organen von normalen und $\mathrm{Mg}$-arm ernährten Ratten. Angaben in $\mu$ Mol/Min. $\cdot g$ Feuchtgewicht (U/g)

\begin{tabular}{|c|c|c|c|c|c|c|c|c|}
\hline & \multicolumn{2}{|c|}{ Niere } & \multicolumn{2}{|c|}{ Herz } & \multicolumn{2}{|c|}{ Leber } & \multicolumn{2}{|c|}{ Muskel } \\
\hline & Kontrolle & Mg-arm & Kontrolle & Mg-arm & Kontrolle & Mg-arm & Kontrolle & Mg-arm \\
\hline HEX & $0,61 \pm 0,04$ & $0,62 \pm 0,04$ & $0,39 \pm 0,03$ & $0,49 \pm 0,03$ & $0,17 \pm 0,03$ & $0,12 \pm 0,02$ & $0,09 \pm 0,01$ & $0,13 \pm 0,01$ \\
\hline PFK & $0,07 \pm 0,02$ & $0,07 \pm 0,02$ & $3,58 \pm 0,71$ & $2,55 \pm 0,30$ & $0,09 \pm 0,03$ & $0,07 \pm 0,02$ & $0,08 \pm 0,02$ & $0,15 \pm 0,02$ \\
\hline ALD & $16,8 \pm 1,5$ & $17,0 \pm 1,1$ & $7,18 \pm 0,34$ & $6,57 \pm 0,34$ & $2,61 \pm 0,17$ & $3,20 \pm 0,24$ & $60,6 \pm 6,0$ & $41,2 \pm 3,4$ \\
\hline ENOL & $48,7 \pm 1,7$ & $45,1 \pm 6,0$ & $30,9 \pm 1,80$ & $26,3 \pm 1,85$ & $19,6 \pm 0,9$ & $28,0 \pm 1,3$ & $137,0 \pm 10$ & $107 \pm 9,0$ \\
\hline PK & $17,0 \pm 2,5$ & $18,6 \pm 1,0$ & $64,8 \pm 5,1$ & $78,2 \pm 3,7$ & $14,7 \pm 1,9$ & $21,1 \pm 2,1$ & $204 \pm 14$ & $164 \pm 12$ \\
\hline LDH & $142 \pm 9,5$ & $153 \pm 6,4$ & $453 \pm 10,3$ & $461 \pm 14,2$ & $575 \pm 40$ & $685 \pm 42$ & $710 \pm 30$ & $715 \pm 55$ \\
\hline G-6-PDH & $1,13 \pm 0,05$ & $1,28 \pm 0,07$ & $0,20 \pm 0,02$ & $0,29 \pm 0,03$ & $2,18 \pm 0,19$ & $4,30 \pm 0,54$ & $0,04 \pm 0,01$ & $0,07 \pm 0,01$ \\
\hline GIDH & $14,1 \pm 1,0$ & $14,1 \pm 0,5$ & $2,11 \pm 0,09$ & $2,32 \pm 0,12$ & $0,76 \pm 0,09$ & $0,73 \pm 0,04$ & $0,15 \pm 0,03$ & $0,16 \pm 0,01$ \\
\hline GPT & $6,80 \pm 0,78$ & $5,47 \pm 0,84$ & $2,72 \pm 0,12$ & $2,91 \pm 0,12$ & $7,10 \pm 0,42$ & $6,42 \pm 0,55$ & $1,23 \pm 0,09$ & $1,15 \pm 0,12$ \\
\hline GOT & $72,4 \pm 4,4$ & $66,0 \pm 3,9$ & $112,5 \pm 3,9$ & $118,5 \pm 5,0$ & $41,5 \pm 1,4$ & $57,0 \pm 4,2$ & $21,7 \pm 2,2$ & $22,9 \pm 2,1$ \\
\hline ICDH & $9,70 \pm 0,47$ & $11,6 \pm 0,86$ & $23,2 \pm 1,8$ & $27,0 \pm 2,1$ & $8,42 \pm 0,48$ & $8,40 \pm 0,45$ & $1,35 \pm 0,20$ & $1,44 \pm 0,16$ \\
\hline
\end{tabular}

wir in der Leber, die Zunahme der Glucose-6-phosphatdehydrogenase-Aktivität in der Niere war allerdings nicht signifikant. Auch die anderen Enzymaktivitäten in der Niere zeigten keine signifikanten Unterschiede. Offenbar kompensieren sich die histochemisch in verschiedenen Nierenabschnitten feststellbaren $\mathrm{Zu}-$ und Abnahmen einiger Dehydrogenase-Aktivitäten (6).

Darüberhinaus waren im Herzmuskel die Aktivität der Pyruvatkinase und in der Leber die Aktivität der Enolase, Pyruvatkinase und Aspartattransaminase erhöht, in der Skelettmuskulatur die Aktivität der Aldolase, der Enolase und der Pyruvatkinase vermindert. In diesem Zusammenhang sei erwähnt, daß eine Erhöhung der Ornithintranscarbamylase (EC 2.1.3.3) in der Leber von Mg-arm ernährten Tieren beschrieben wurde (7).

Die Veränderungen der Enzymaktivitäten waren gering und betrugen maximal das 0,5 - bzw. 2 fache der Kontrollwerte.

Die Ursachen für das Verhalten der Enzymaktivitäten sind nicht bekannt. Es ist formal möglich, daß sich die Synthese- oder die Abbaugeschwindigkeiten bei einigen Enzymen im $\mathrm{Mg}$-Mangelzustand geändert haben. $\mathrm{Ob}$ hierfür die geringfügigen Änderungen des intrazellulären $\mathrm{Mg}$-, $\mathrm{Na}$-, $\mathrm{K}$ - und $\mathrm{Ca}$-Gehaltes unmittelbar verantwortlich sind, bleibt offen, ebenso, ob sich Konsequenzen für den Zellstoffwechsel ergeben.

Von den getesteten Enzymen, die in der Zelle die höchsten Aktivitäten haben, bestimmten wir zusätzlich ihre Aktivität im Serum (Tab. 2). Dabei fanden wir, daß im Serum der Mg-arm ernährten Ratten die Enolase-Aktivität vermindert, die Lactatdehydrogenase-Aktivität un-
Tab. 2

Enzymaktivitäten im Serum normaler und Mg-arm ernährter Ratten. Angaben in $\mathrm{nMol} / \mathrm{Min} . \cdot \mathrm{ml}(\mathrm{mU} / \mathrm{ml})$

\begin{tabular}{lrc}
\hline & Kontrolle & Mg-arm \\
\hline ENOL & $40,1 \pm 2,4$ & $27,3 \pm 2,7$ \\
PK & $6,0 \pm 1,2$ & $19,4 \pm 2,4$ \\
LDH & $206 \pm 12$ & $200 \pm 7$ \\
GOT & $77,5 \pm 6,6$ & $149,5 \pm 15,7$ \\
\hline
\end{tabular}

verändert und die Pyruvatkinase- sowie Aspartattransaminase-Aktivität erhöht waren. Eine erhöhte SerumAspartattransaminase und eine unveränderte Lactatdehydrogenase-Aktivität im Serum von $\mathrm{Mg}$-arm ernährten Ratten wurde inzwischen auch von KIESEL und Mitarbeitern (8) beschrieben. Darüber hinaus war im Serum von $\mathrm{Mg}$-Mangeltieren die Aktivität der alkalischen Phosphatase angestiegen, die Aktivität der Alanintransaminase, Isocitratdehydrogenase, der sauren Phosphatase und der $\beta$-Hydroxy-buttersäuredehydrogenase gleichgeblieben und die Aldolase-Aktivität abgesunken (8).

Als Ursachen für die veränderten Enzymaktivitäten im Serum kann man eine veränderte Permeabilität und/oder eine erhöhte Absterberate von Zellen bei einer für die einzelnen Enzyme verschiedenen Eliminationsgeschwindigkeit aus dem Serum (9) annèhmen. Damit übëreinstimmend sieht man in den elektronenmikroskopischen Bildern (10) von Niere, Herz und Muskel Mg-arm ernährter Tiere freie Zellorganellen zwischen den Zellen. Die dazugehörenden Zellen können in vivo oder während der Präparation zerfallen sein. Dieser Befund zeigt jedoch, daß die Zellmembranen bei $\mathrm{Mg}$-arm ernährten Tieren weniger stabil sind.

\section{Literatur}

1. Günther, Th. und P. Mariss, Zschr. Naturforsch. $23 b, 338$ (1968). - 2. GüNTHER, TH., Zschr. Naturforsch. 24b, 428 (1969). 3. GüNTher, Th., diese Z. 8, 65 (1970). - 4. v. FeLLENBERG, R., H. Eppenterger, R. Richterich und H. Aebi, Biochem. Z. 336, 334 (1962). - 5. Zitiert nach 4. - 6. Hess, R., I. MAC INTYRE, N. Alcock und A. G. E. Pearse, Brit. J. Exper. Path. 40, 80 (1959).
7. Lizzarratde, G., V. E. Mazzocco und E. B. Flink, Proc. Soc. Exper. Biol. Med. 126, 249 (1967). - 8. Kreser, G. K., H. D. Alexander und G. Brooks, Cornell Vet. 59,89 (1969). - 9. AMELUNG, D., Hoppe-Seyler's Z. physiol. Chem. 318, 219 (1960). 10. Merker, H. J. und Th. GüNTher, diese Z. 8, 71 (1970). 